OnLine Journal of Biological Sciences 9 (2): 52-61, 2009

ISSN 1608-4217

(C) 2009 Science Publications

\title{
Four Dimensional (4-D) BioChemInfoPhysics Models of Cardiac Cellular and Sub-Cellular Vibrations (Oscillations)
}

\author{
Kang Cheng and Chang-Hua Zou \\ Science Research, 205 Hana Road, Edison NJ 08817, USA
}

\begin{abstract}
Problem statement: Cardiovascular Diseases (CVD) continued to be the leading cause of death. Failure or abnormal cardiac cellular or sub-cellular vibrations (oscillations) could lead failure or abnormal heart beats that could cause CVD. Understanding the mechanisms of the vibrations (oscillations) could help to prevent or to treat the diseases. Scientists have studied the mechanisms for more than 100 years. To our knowledge, the mechanisms are still unclear today. In this investigation, based on published data or results, conservation laws of the momentum as well as the energy, in views of biology, biochemistry, informatics and physics (BioChemInfoPhysics), we proposed our models of cardiac cellular and sub-cellular vibrations (oscillations) of biological components, such as free ions in Biological Fluids (BF), Biological Membranes (BM), $\mathrm{Ca}++\mathrm{H}+(\mathrm{Ca}++$ and $\mathrm{Na}+\mathrm{K}+)$ ATPases, $\mathrm{Na}+\mathrm{Ca}++$ exchangers (NCX), Ca++ carriers and myosin heads. Approach: Our models were described with 4-D $(\mathrm{x}, \mathrm{y}, \mathrm{z}, \mathrm{t}$ or $\mathrm{r}, \theta, \mathrm{z}, \mathrm{t})$ momentum transfer equations in mathematical physics. Results: The momentum transfer equations were solved with free and forced, damped, un-damped and over-damped, vibrations (oscillations). The biological components could be modeled as resonators or vibrators (oscillators), such as liquid plasmas, membranes, active springs, passive springs and active swings. Conclusion: We systematically provided new insights of automation (ignition and maintain), transportation, propagation and orientation of the cardiac cellular and sub-cellular vibrations (oscillations) and resonances, with our BioChemInfoPhysics models of 4-D momentum transfer equations. Our modeling results implied: Autorhythmic cells (Sinoatrial Node Cells (SANC), Atrioventricular Node Cells (AVNC), Purkinje fibers), non-Auto-rhythmic ventricular myocytes and their Sarcoplasmic Reticulums (SR) work as Biological Liquid Plasma Resonators (BLPR). The resonators were biological clocks and mainly made of BF, BM and BM Transporters (BMT) that had mutually adapted and produced Biological Liquid Plasma Resonance Frequencies (BLPRF) for the resonators during their natural evolution. The resonators naturally vibrate (oscillate) near the SANC SR BLPRF that had the highest BLPRF among them.
\end{abstract}

Key words: Momentum, plasma, resonator, spring, swing, membrane, ATPase, NCX, carrier, myosin

\section{INTRODUCTION}

Cardiovascular Diseases (CVD) continued to be the leading cause of death, an estimated 80 millions American adults (approximately 1 in 3 ) had one or more types of CVD in $2005^{[1]}$. Failure or abnormal cardiac cellular or sub-cellular vibrations (oscillations) could lead failure or abnormal heart beats that could cause CVD. Understanding the mechanisms of the vibrations (oscillations) could help to prevent or to treat the diseases. Scientists have studied the mechanisms for more than 100 years ${ }^{[2,3]}$ and proposed models for the mechanisms. The models respectively involved mathematics $^{[4,5]}$, biophysics ${ }^{[6,7]}$, biochemistry ${ }^{[8-18]}$. But, none involved the automation of the vibrations (oscillations) ${ }^{[2]}$. To our knowledge, the mechanisms are still unclear today.
In this investigation, based on published data or results ${ }^{[2-18]}$, conservation laws of the momentum as well as the energy, in views of biology, biochemistry, informatics and physics (BioChemInfoPhysics), we proposed models of cardiac cellular and sub-cellular vibrations (oscillations) of biological components, such as free ions in Biological Fluids (BF), Biological Membranes (BM), $\mathrm{Ca}++\mathrm{H}+(\mathrm{Ca}++$ and $\mathrm{Na}+\mathrm{K}+)$ ATPases, $\mathrm{Na}+\mathrm{Ca}++$ exchangers (NCX), $\mathrm{Ca}++$ carriers and myosin heads. Moreover, we discuss our models' general applications and general meanings in biology.

\section{MATERIALS AND METHODS}

Model developments: Based on the published experimental results ${ }^{[2-18]}$, we approximately consider, $\mathrm{BF}$ in SR, cells or extra-cells, as quasi liquid plasmas in

Corresponding Author: Kang Cheng, Science Research, 205 Hana Road, Edison NJ 08817, USA Tel/Fax: 732-248-0790 
physics, the plasmas are quasi neutral electromagnetically; biological or biochemical components such as free $\mathrm{Ca}++$ in BF, SR SubMembrane (SRSM) or Cell Membrane (CM), $\mathrm{Ca}++\mathrm{H}+$ $(\mathrm{Ca}++$ or $\mathrm{Na}+\mathrm{K}+)$ ATPases, $\mathrm{NCX}, \mathrm{Ca}++$ carriers and myosin heads, because disturbances, internal excitations or external stimulations always exist, often are displaced from their equilibrium positions or distances, the redistribution of charges and masses will respectively set up forces, tensions or pressures at the components. The forces, the tensions or the pressures will restore the components back to their original equilibrium positions or distances and could initiate a vibration (oscillation), even a resonance. Under some conditions, the vibration (oscillation), even the resonance continues as long as an internal excitation or an external stimulation is powerful enough and retained.

Longitudinal vibrations (oscillations) of free ions in BF: Based on published biological and biochemical experimental results ${ }^{[2-3,9-10]}$, the above analysis, the liquid plasma physics ${ }^{[19]}$, electrodynamics and hydrodynamics, we propose our model of longitudinal vibrations (oscillations) of free ions in BF, e.g., ions in SR, Cellular Fluid (CF) or Extra Cellular Fluid (ECF) and i.e., directions of the vibrations (oscillations) and the waves propagations are parallel from each other. Figure 1, we consider the SR and the cells as BLPR with $\mathrm{Ca}++$. The momentum transfer equation of the model is:

$\frac{d}{d t}\left(v_{i} m_{i} n_{i}\right)=-\nabla P_{i}-v_{i} m_{i} n_{i} c_{i}+q_{i} n_{i}\left(E+v_{i} X B\right)+F_{v}$

Where:

$\mathrm{v}_{\mathrm{i}}, \mathrm{m}_{\mathrm{i}}, \mathrm{n}_{\mathrm{i}}, \mathrm{c}_{\mathrm{i}}$ and $\mathrm{q}_{\mathrm{i}}=$ Respectively the velocity, the mass, the particle concentration, the effective collision frequency (Ohm's law related) and the electric charge of the free ions

$\nabla \quad=$ A 3-D spatial Nabla mathematic operator

$\mathrm{P}_{\mathrm{i}} \quad=$ Represents the pressure in the fluid

$\mathrm{E}$ and $\mathrm{B} \quad=$ Electric and magnetic fields (including interactions or couplings) respectively

$\mathrm{X}=$ Means a mathematical cross product

$\mathrm{F}_{\mathrm{v}} \quad=\mathrm{A}$ sum of externally applied hydrodynamic volume forces (force/volume) (including interactions or couplings)

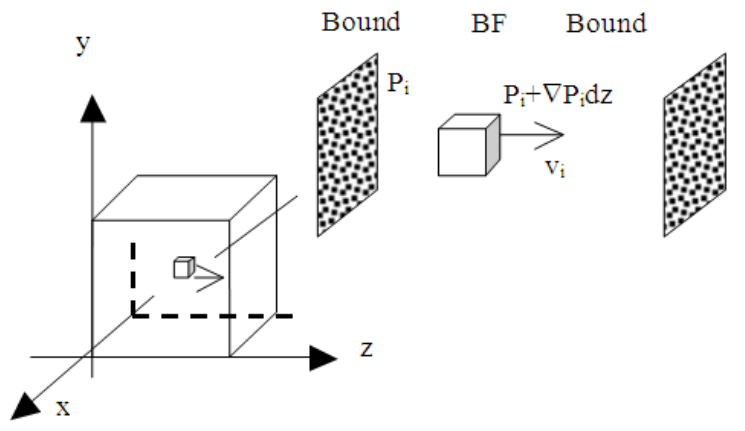

Fig. 1: A unit volume of free ions vibrates (oscillates) in BLPR. BLPR are SR or cells, the bounds are $\mathrm{BM}$, such as SRSM or CM in Fig. 1. The ions are assumed to vibrate (oscillate) in $\mathrm{z}$ direction to simplify the illustration

Besides:

$\nabla \bullet\left(\mathrm{v}_{\mathrm{i}} \mathrm{m}_{\mathrm{i}} \mathrm{n}_{\mathrm{i}}\right)=\mathrm{m}_{\mathrm{i}}\left(\mathrm{g}_{\mathrm{i}} \mathrm{n}_{\mathrm{i}}-\frac{\partial \mathrm{n}_{\mathrm{i}}}{\partial \mathrm{t}}\right)$

Where:

$\nabla \bullet=$ Means a 3-D spatial divergence, a mathematical operator

$\mathrm{g}_{\mathrm{i}}=\mathrm{A}$ hydrodynamic growth factor ${ }^{[20]}$ of ions, or a regeneration (recombination) factor of ions, e.g., a factor to evaluate $\mathrm{Ca}++$ binding or leaving troponins

For alternating currents or releases of natural biological ions, we neglect the magnetic force and Column's field

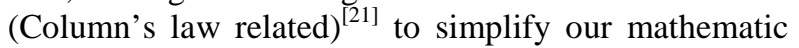
process. Therefore, from Eq. 1, removing the last three terms at the right side, applying divergence to both sides, interchanging divergent and derivative operations at the left side; using Eq. 2, considering actual liquid with a compressibility ${ }^{[22]}$, using the partial differentiation of time to approximate the differentiation of time and modifying a published assumption $^{[23]}, \mathrm{P}_{\mathrm{i}}=\mathrm{V}_{\mathrm{i}}^{2} \mathrm{~m}_{\mathrm{i}} \mathrm{n}_{\mathrm{i}}$ ( $\mathrm{Vi}$ is an averaged velocity of the free ions), we obtain a correspondent homogeneous wave equation:

$\left[\frac{\partial^{2} n_{i}}{\partial t^{2}}-\left(g_{i}+c_{i}\right) \frac{\partial n_{i}}{\partial t}+c_{i} g_{i} n_{i}\right] \frac{1}{V_{i}^{2}}=\nabla^{2} n_{i}$

where, $\nabla^{2}$ means a 3-D spatial Laplace mathematical operator.

Transverse vibrations (oscillations) of BM: Based on published biological and biochemical experimental 
results $^{[2,3,12]}$, the above analysis, the membrane vibration theory ${ }^{[23]}$, electrodynamics, we propose our model of transverse vibrations (oscillations) of BM, e.g., SRSM or CM and i.e., directions of the vibrations (oscillations) and the waves propagations are perpendicular from each other. Figure 2, we consider a piece of membrane transversely vibrates (oscillates) on the surfaces of SR or cells that are modeled as rectangular BLPR and assume the membrane surfaces are in $\mathrm{x}, \mathrm{y}$ and $\mathrm{z}$ planes respectively; and vibrations (oscillations) directions are mostly in $\mathrm{x}, \mathrm{y}$ and $\mathrm{z}$ directions respectively. The momentum transfer equation of the model for a piece of membrane in $\mathrm{z}$ planes is:

$$
\begin{aligned}
& \rho_{\mathrm{mm}} \frac{\partial^{2} \mathrm{z}_{\mathrm{m}}}{\partial \mathrm{t}^{2}}+v_{\mathrm{mz}} \frac{\partial \mathrm{z}_{\mathrm{m}}}{\partial \mathrm{t}}-\mathrm{T}_{\mathrm{mz}}\left(\frac{\partial^{2} \mathrm{z}_{\mathrm{m}}}{\partial \mathrm{x}^{2}}+\frac{\partial^{2} \mathrm{z}_{\mathrm{m}}}{\partial \mathrm{y}^{2}}\right) \\
& =\rho_{\mathrm{mc}}\left(\mathrm{E}_{\mathrm{z}}+\frac{\partial \mathrm{z}_{\mathrm{m} \perp}}{\partial \mathrm{t}} \mathrm{XB}_{\mathrm{z} \perp}\right)+P_{\mathrm{mz}}
\end{aligned}
$$

Where:

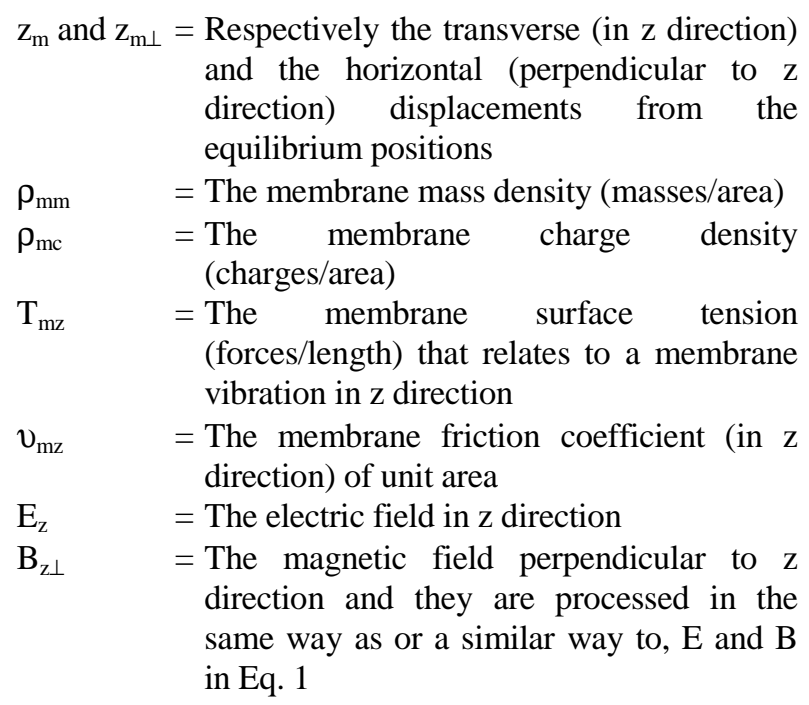

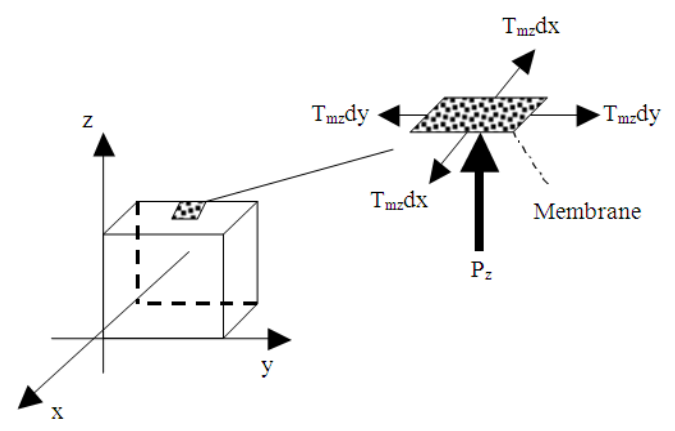

Fig. 2: A piece of membrane vibrates (oscillates) on surfaces of BLPR. BLPR are SR or cells, the surfaces are BM, such as SRSM or CM in Fig. 2. $\mathrm{Pz}$ is a sum of pressures in $\mathrm{z}$ direction
But, both Coulomb's filed and Ohm's field play roles across the membranes. $\mathrm{P}_{\mathrm{mz}}$ represents a sum of externally applied mechanic or hydrodynamic pressures (forces/area) (including interactions or couplings) in $\mathrm{z}$ direction. We can respectively obtain the momentum transfer equations for that on $\mathrm{x}$ and $\mathrm{y}$ planes by rotating $\mathrm{z}, \mathrm{x}$ and $\mathrm{y}$ in Eq. 4.

Ca++H+ ATPase vibrations (oscillations): We model a section about the $\mathrm{Ca}++$ binding sites and the (aspartic acid) phosphorylation site of the ATPase as a spring, with some charges, an effective mass and an effective stiffness, based on the published biochemical data $^{[2,3,8,13-15]}$, conservation laws of the (angular) momentum as well as the energy, the vibration theory $^{[24]}$ and electrodynamics. Figure 3 show our model in BioChemInfoPhysics rather than presents a structure model in molecular biology. The both (angular) momentum and energy are conserved in each procedure in the Fig. 3. Beside the states of open and close for gates of ions transportations, we define a high impedance state as that only ions with a high kinetic energy can go through the gates, most thermal motion ions can not pass the gates. We consider only working $\mathrm{Ca}++\mathrm{H}+\mathrm{ATPases}$ that are able to eject $\mathrm{Ca}++$ into SR. We assume, the spring model is across SRSM in $\mathrm{z}$ planes and performs a 4-D (r, $\theta, \mathrm{z}, \mathrm{t})$ vibrations (oscillations) in a cylindrical coordinates system; $\mathrm{z}$ direction vibration (oscillation) periodically and mostly provide the power to eject $\mathrm{Ca}++$ into $\mathrm{SR} ; \mathrm{z}_{\mathrm{s} 0}$ represents an averaged relative equilibrium distance in $\mathrm{z}$ direction between the $\mathrm{Ca}++(\mathrm{H}+)$ binding sites and the phosphorylation site; $\mathrm{z}_{\mathrm{s}}$ represents a displacement from the averaged relative equilibrium distance; vibrations in $\mathrm{r}$ and $\theta$ directions will periodically and mainly open, close or set a high impedance to the three states of gates. (a) Two $\mathrm{Ca}++$ and one ATP are binding a vibrating $\mathrm{Ca}++\mathrm{H}+$ ATPase when the $\mathrm{Ca}++$ gate facing the cellular is opened. The information of the binding sites is stored as charges, transmitted with the electromagnetic fields, recognized and responded by Lorentz forces $^{[25]}$ in a range about from 0.3-3 nm. Some binding energy is released during the bindings ${ }^{[3]}$. (b) After the bindings, the ATPase's conformation is changed, the ATP binding site with the ATP moves, from its equilibrium position, to a position that is more proximate to the phosphorylation site and orientates the site more in $\mathrm{z}$ direction. The spring contracts more than the free state and so that more potential energy is stored. The $\mathrm{Ca}++$ gate facing the cellular is closed. The ATPase is hydrolyzing and breaking the bound ATP into ADP and P group, because of the electric forces mostly in $\mathrm{z}$ direction. 

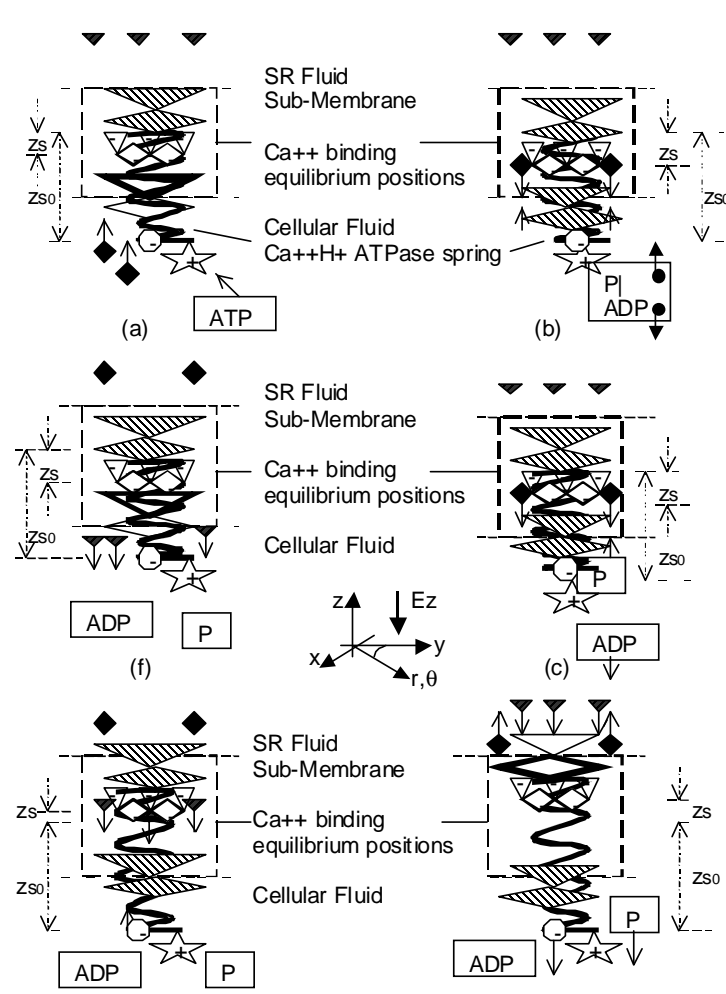

(e)

(d)

$\mathrm{Ca}++$ : ion, binding site, gate $(\mathrm{O}, \mathrm{C}, \mathrm{H})$ $\mathrm{H}+$ : ion, binding site, gate $(\mathrm{O}, \mathrm{C}, \mathrm{H})$

ATP binding site: $\approx$ s phosphorylation site: $\bigcirc$ moving direction: $<$ breaking forces: $\rightarrow$

Fig. 3: Our BioChemInfoPhysics (spring) model of a working $\mathrm{Ca}++\mathrm{H}+$ ATPase shows a cycle of $\mathrm{Ca}++/ \mathrm{H}+$ transportations across SRSM. Three states of the gates: $\mathrm{O}=$ Open; $\mathrm{C}=$ Close; $\mathrm{H}=$ High impedance. $\mathrm{Ez}$ is an assumed electric field across SRSM

(c) After the breaking, the released high hydrolyzed energy (from $11-13 \mathrm{kcal} \mathrm{mole}^{-1}$, about $0.5 \mathrm{eV}$ ) ${ }^{[3]}$ is converted to the kinetic energies of the ADP and the $\mathrm{P}$ group, the ADP and the $\mathrm{P}$ group move in opposite directions. The ADP is released and the $\mathrm{P}$ group hits the phosphorylation site first, then hits somewhere near to the $\mathrm{Ca}++$ binding sites and transfers its momentum and kinetic energy to the $\mathrm{Ca}++$ and the spring, more potential energy is stored than the free state. (d) The spring finishes the contraction. Then it is extending. The Ca++ gate facing SR is at a high impedance state. If the kinetic energy of the $\mathrm{Ca}++$ is greater than the bond energy, the $\mathrm{Ca}++$ is being ejected to the SR. The $\mathrm{P}$ group responds from the spring. The $\mathrm{H}+$ gate facing $\mathrm{SR}$ is opened and three $\mathrm{H}+$ are going to bind the ATPase. The ATP binding site returns to its equilibrium position. (e) After the $\mathrm{Ca}++$ are ejected to SR and the three $\mathrm{H}+$ bind the sites, the $\mathrm{Ca}++$ gate facing SR and the $\mathrm{H}+$ gate facing SR are closed. The spring contracts. (f) The $\mathrm{H}+$ gate facing the cellular is at a high impedance state. The contracting potential energy is converted to the kinetic energies of the $\mathrm{H}+$ and the spring and some others. If the kinetic energy of $\mathrm{H}+$ is greater than the bond energy, the $\mathrm{H}+$ is being ejected to the cellular. The $\mathrm{Ca}++$ gate facing the cellular is opened again to complete the cycle. If no $\mathrm{H}+$ is transported, no $\mathrm{H}+$ activities are in procedures (e) and (f).

Based on the above analysis, we propose our momentum transfer equation for the spring models across SRSM in z planes:

$$
\mathrm{m}_{\mathrm{sz}} \frac{\mathrm{d}^{2} \mathrm{z}_{\mathrm{s}}}{\mathrm{dt}^{2}}+\mathrm{v}_{\mathrm{sz}} \frac{\mathrm{dz}_{\mathrm{s}}}{\mathrm{dt}}+\mathrm{k}_{\mathrm{sz}} \mathrm{z}_{\mathrm{s}}=\sum_{1} \mathrm{q}_{\mathrm{sl}}\left(\mathrm{E}_{\mathrm{z}}+\frac{\mathrm{dz}_{\mathrm{s} \perp}}{\mathrm{dt}} \mathrm{XB}_{\mathrm{z \perp}}\right)+\mathrm{F}_{\mathrm{sz}}
$$

Where:

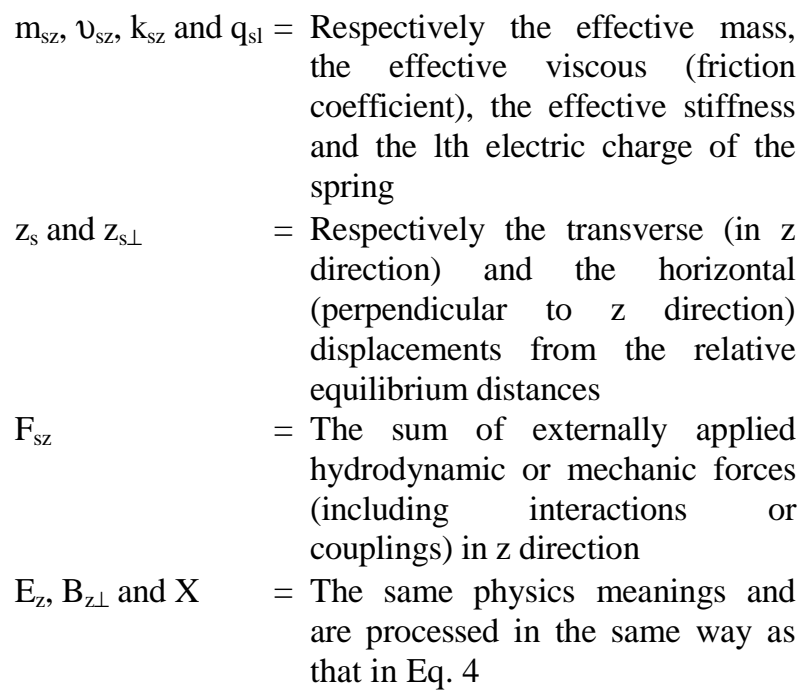

From Eq. 5, we can obtain vibrating equations, in $\mathrm{r}$ direction by changing $\mathrm{z}$ to $\mathrm{r}$ and in $\theta$ direction by changing $\mathrm{z}$ to $\theta, \mathrm{m}$ to $\mathrm{I}$ and cross multiplying $\mathrm{r}$ at the right side of Eq. 5 to obtain torques (general forces), where I means a moment of inertial (a general mass) and we can also respectively obtain the momentum transfer equations for that in $\mathrm{x}$ and $\mathrm{y}$ planes by rotating $\mathrm{z}, \mathrm{x}$ and $\mathrm{y}$.

$\mathrm{Na}+\mathrm{K}+$ ATPase vibrations (oscillations): Because biological and biochemical experimental data have shown that ${ }^{[13,14,22-16]}, \mathrm{Na}+\mathrm{K}+$ ATPase has a very similar structure to that of $\mathrm{Ca}++\mathrm{H}+$ or $\mathrm{Ca}++$ ATPase, our model for the $\mathrm{Na}+\mathrm{K}+$ ATPase is, the same as (Eq. 5), in physics and informatics, or similar to (Fig. 3), in 
biology and biochemistry, that for $\mathrm{Ca}++\mathrm{H}+$ ATPase. The major difference is, a $\mathrm{Na}+\mathrm{K}+$ ATPase transports three $\mathrm{Na}+$ to the extra-cellular and two $\mathrm{K}+$ to the cellular.

NCX vibrations (oscillations): In the same way (Eq. (5)), in physics and informatics, as that of proposing and developing the model for $\mathrm{Ca}++\mathrm{H}+$ ATPases vibrations (oscillations), we propose and develop our model for a working NCX vibrations (oscillations), based on the published experimental biological and biochemical data ${ }^{[2,3,17]}$. The main differences from a $\mathrm{Ca}++\mathrm{H}+$ ATPases are: (1) a NCX transports three $\mathrm{Na}+$ to the cellular (or the extra-cellular) and one $\mathrm{Ca}++$ to the extra-cellular (or the cellular); (2) an exchanger does not cost any biological energy directly. The main source of driving forces are the electric field and the thermal collision, Fig. 4. Where, $z_{s}$ represents a displacement of the $\mathrm{Ca}++$ binding site from the site's equilibrium position.

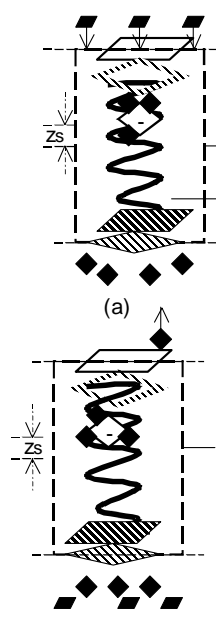

(f)

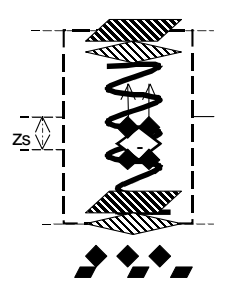

(e)

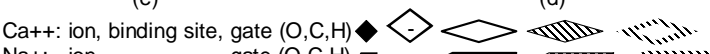

$\mathrm{Na}+$ : ion,

moving direction: $\longleftarrow$

Fig. 4: Our BioChemInfoPhysics (spring) model of a working NCX shows a cycle of $\mathrm{Na}+\mathrm{Ca}++$ transportations across CM. Only one $\mathrm{Ca}++$ binding site is presented to illustrate the model clearer. Three states of the gates: $\mathrm{O}=$ Open; $\mathrm{C}=$ Close; $\mathrm{H}=$ High impedance
Ca++ carrier vibrations (oscillations): In the same way (Eq. 5), in physics and informatics, as that of proposing and developing the model for $\mathrm{Ca}++\mathrm{H}+$ ATPases vibrations (oscillations), we propose and develop our model for a working $\mathrm{Ca}++$ carrier vibrations (oscillations), based on the published experimental biological and biochemical data ${ }^{[3]}$. The main differences from a $\mathrm{Ca}++\mathrm{H}+$ ATPases are (1) A carrier transports the same ions across a membrane in two opposite directions, the net transportation is dependent on the ions concentrations and the electromagnetic fields; (2) A carrier does not cost any biological energy directly. The main source of driving forces are the electric field and the thermal collision, Fig. 5. Where, $z_{\mathrm{s}}$ represents a displacement of the $\mathrm{Ca}++$ binding site from the site's equilibrium position.

Myosin head vibrations (oscillations): In a similar way (Eq. 5), in physics and informatics, to that of proposing and developing the model for $\mathrm{Ca}++\mathrm{H}+$ ATPases vibrations (oscillations), we propose and develop our model of working myosin head vibrations, based on the published biological and biochemical data ${ }^{[3]}$.
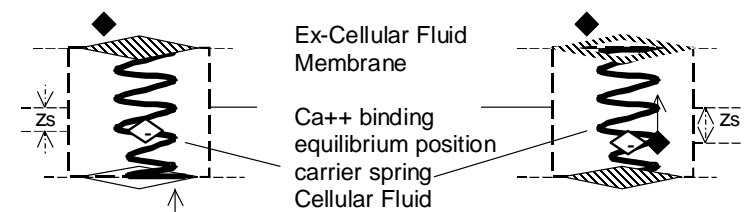
Cellular Fluid (a)

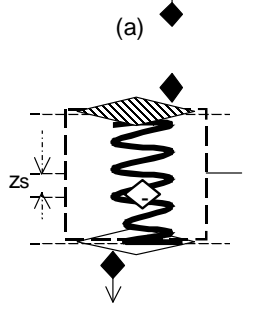

(e)

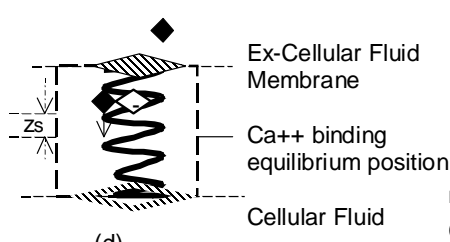

(d)

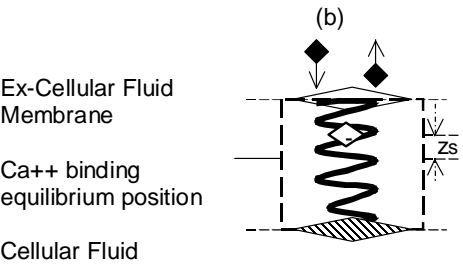

(c)

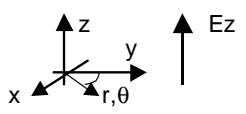

moving direction: $\mathrm{Ca}++$ ion: Ca++ binding site: $\diamond$

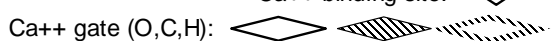

Fig. 5: Our BioChemInfoPhysics (spring) model of a working $\mathrm{Ca}++$ carrier shows a cycle of $\mathrm{Ca}++$ transportation across CM. Three states of the gates: $\mathrm{O}=$ open; $\mathrm{C}=$ close; $\mathrm{H}=$ high impedance. $\mathrm{Ez}$ is an assumed electric field across the membrane 
OnLine J. Biol. Sci., 9 (2): 52-61, 2009

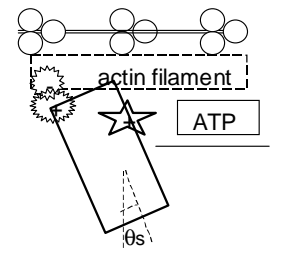

(a)

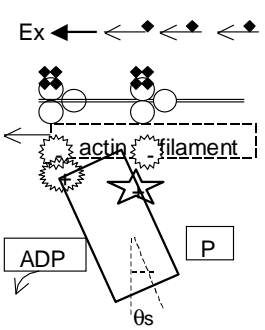

(f)

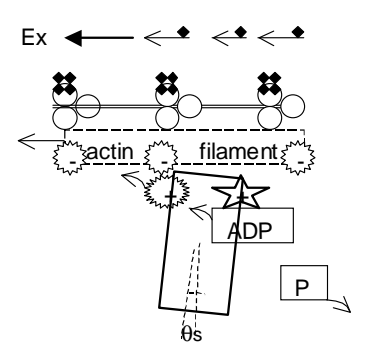

(e)

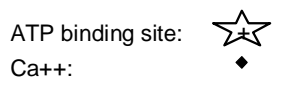

moving direction:

Fig. 6: Our BioChemInfoPhysics (swing) model of a working myosin head shows a cycle of myosinactin stroking. Ex is the Ohm's electric field

The main differences from a $\mathrm{Ca}++\mathrm{H}+\mathrm{ATPases}$ are: (1) The vibration (oscillation) model is a swing rather than a spring, torques and a moment of inertial play major roles to stroke actins; (2) Ohm's field plays roles in the $\mathrm{BF}$; (3) the $\mathrm{P}$ group is released first and the ADP is released second Fig. 6.

\section{RESULTS}

For Eq. 1, 4 and 5, we assume, the vibrations (oscillations) are spatially independent from each other and temporally synchronous to obtain mathematical and analytical solutions $^{[24]}$.

Solutions of longitudinal vibrations (oscillations) of free ions in BF: For the homogeneous Eq. 3, free vibrations (oscillations), using separation of variables [23], we obtain solutions:

$$
\begin{aligned}
& \mathrm{n}_{\mathrm{ih}}(\mathrm{x}, \mathrm{y}, \mathrm{z}, \mathrm{t})=\mathrm{X}_{\mathrm{ih}}(\mathrm{x}) \mathrm{Y}_{\mathrm{ih}}(\mathrm{y}) \mathrm{Z}_{\mathrm{ih}}(\mathrm{z}) \mathrm{T}_{\mathrm{ih}}(\mathrm{t}) \\
& \mathrm{X}_{\mathrm{ih}}(\mathrm{x})=\mathrm{A}_{\mathrm{ixh}} \exp \left(\mathrm{ik}_{\mathrm{ixh}} \mathrm{x}\right)+\mathrm{B}_{\mathrm{ixh}} \exp \left(-\mathrm{ik}_{\mathrm{ixh}} \mathrm{x}\right) \\
& \mathrm{Y}_{\mathrm{ih}}(\mathrm{y})=\mathrm{A}_{\mathrm{iyh}} \exp \left(\mathrm{ik}_{\mathrm{iyh}} \mathrm{y}\right)+\mathrm{B}_{\mathrm{iyh}} \exp \left(-\mathrm{ik}_{\mathrm{iyh}} \mathrm{y}\right) \\
& \mathrm{Z}_{\mathrm{ih}}(\mathrm{z})=\mathrm{A}_{\mathrm{izh}} \exp \left(\mathrm{ik}_{\mathrm{izh}} \mathrm{z}\right)+\mathrm{B}_{\mathrm{izh}} \exp \left(-\mathrm{ik}_{\mathrm{izh}} \mathrm{z}\right) \\
& \mathrm{T}_{\mathrm{ih}}(\mathrm{t})=\mathrm{A}_{\mathrm{ih}} \exp \left[\left(\alpha_{\mathrm{ih}}+\beta_{\mathrm{ih}}\right) \mathrm{t}\right]+\mathrm{B}_{\mathrm{ih}} \exp \left[\left(\alpha_{\mathrm{ih}}-\beta_{\mathrm{ih}}\right) \mathrm{t}\right] \\
& \mathrm{k}_{\mathrm{ih}}^{2}=\mathrm{k}_{\mathrm{ixh}}^{2}+\mathrm{k}_{\mathrm{iyh}}^{2}+\mathrm{k}_{\mathrm{izh}}^{2} \\
& \alpha_{\mathrm{ih}}=\frac{\mathrm{g}_{\mathrm{i}}-\mathrm{c}_{\mathrm{i}}}{2} \\
& \beta_{\mathrm{ih}}=\frac{\sqrt{\left(\mathrm{g}_{\mathrm{i}}-\mathrm{c}_{\mathrm{i}}\right)^{2}+4\left(\mathrm{c}_{\mathrm{i}} \mathrm{g}_{\mathrm{i}}-\mathrm{V}_{\mathrm{i}}^{2} \mathrm{k}_{\mathrm{ihh}}^{2}\right)}}{2}
\end{aligned}
$$

$\mathrm{A}_{\mathrm{ixh}}, \mathrm{B}_{\mathrm{ixh}}, \mathrm{A}_{\mathrm{iyh}}, \mathrm{B}_{\mathrm{iyh}}, \mathrm{A}_{\mathrm{izh}}, \mathrm{B}_{\mathrm{izh}}, \mathrm{A}_{\mathrm{ih}}, \mathrm{B}_{\mathrm{ih}}$ and $\mathrm{k}_{\mathrm{ixh}}, \mathrm{k}_{\mathrm{iyh}}, \mathrm{k}_{\mathrm{izh}}$, $\mathrm{k}_{\mathrm{ih}}$ (the four are inversely proportional to the resonators' sizes) are constants and they can be obtained with boundary or initial conditions. Let:

$$
\xi_{\mathrm{ih}}=\left(\mathrm{g}_{\mathrm{i}}-\mathrm{c}_{\mathrm{i}}\right)^{2}+4\left(\mathrm{c}_{\mathrm{i}} \mathrm{g}_{\mathrm{i}}-\mathrm{V}_{\mathrm{i}}^{2} \mathrm{k}_{\mathrm{ih}}^{2}\right)
$$

When $\xi_{\text {ih }}<0$ and $\alpha_{\text {ih }}>0$, Eq. 10 represents damped natural vibrations (oscillations), the frequencies are $\mathrm{f}_{\mathrm{in}}=\beta_{\mathrm{ih}} / 2 \pi$. For the cardiac cells and their SR, Ca++ vibrate (oscillate) under the damped mode. For an approximation of free friction and free regeneration, $\xi_{\text {ih }}<0$ and $\alpha_{\text {ih }}=0$, Eq. 10 represents ideal undamped natural vibrations, i.e., simple harmonic oscillations, the frequencies are BLPRF and they are:

$f_{\text {in } 0}=\frac{V_{i} k_{i h}}{2 \pi}$

We believe, for SANC, AVNC, Purkinje fibers and their SR, $\mathrm{Ca}++$ ions could naturally vibrate (oscillate) near the un-damped mode under some conditions. When $\xi_{\text {ih }}>0$, Eq. 10 represents over-damped motions. When $\xi_{\text {ih }}=0$, Eq. 10 represents critical damping motions.

When internal excitations or external stimulations exist, forced vibrations (oscillations) occur. The solutions of Eq. 1 should be mathematically the sum of that for the homogeneous and that for the nonhomogeneous (the forced responses). A resonance occurs when an internal excitation or an external stimulation has a driving (forcing) frequency and a correspondent phase near to the un-damped naturals ${ }^{[24]}$. 
Under resonance, a biological resonator can obtain the optimum responding amplitude Vs energy cost: It can get the maximum biological response amplitude with the same excitation (stimulation) energy cost, or it can get the same biological response amplitude with the minimum excitation (stimulation) energy cost.

Solutions of transverse vibrations (oscillations) of BM: For the free (natural) mechanical vibrations (oscillations) without any internal excitation and external stimulation, Eq. 4 reduces to a homogeneous wave equation after let the terms at right side of the equation be 0 . Assume $\rho_{\mathrm{mm}}$ is a constant and use separation of variables ${ }^{[23]}$, we obtain solutions of the homogeneous equation:

$$
\begin{aligned}
& \mathrm{z}_{\mathrm{mh}}(\mathrm{x}, \mathrm{y}, \mathrm{t})=\mathrm{X}_{\mathrm{mh}}(\mathrm{x}) \mathrm{Y}_{\mathrm{mh}}(\mathrm{y}) \mathrm{T}_{\mathrm{mh}}(\mathrm{t}) \\
& \mathrm{X}_{\mathrm{mh}}(\mathrm{x})=\mathrm{A}_{\mathrm{mxh}} \exp \left(\mathrm{ik}_{\mathrm{mxh}} \mathrm{x}\right)+\mathrm{B}_{\mathrm{mxh}} \exp \left(-\mathrm{ik}_{\mathrm{mxh}} \mathrm{x}\right) \\
& \mathrm{Y}_{\mathrm{mh}}(\mathrm{y})=\mathrm{A}_{\mathrm{myh}} \exp \left(\mathrm{ik}_{\mathrm{myh}} \mathrm{y}\right)+\mathrm{B}_{\mathrm{myh}} \exp \left(-\mathrm{ik} \mathrm{m}_{\mathrm{mh}} \mathrm{y}\right) \\
& \mathrm{T}_{\mathrm{mh}}(\mathrm{t})=\mathrm{A}_{\mathrm{mh}} \exp \left[\left(\alpha_{\mathrm{mh}}+\beta_{\mathrm{mh}}\right) \mathrm{t}\right]+\mathrm{B}_{\mathrm{mh}} \exp \left[\left(\alpha_{\mathrm{mh}}-\beta_{\mathrm{mh}}\right) \mathrm{t}\right] \\
& \mathrm{k}_{\mathrm{mh}}^{2}=\mathrm{k}_{\mathrm{mxh}}^{2}+\mathrm{k}_{\mathrm{myh}}^{2} \\
& \alpha_{\mathrm{mh}}=-\frac{v_{\mathrm{m}}}{2 \rho_{\mathrm{mm}}} \\
& \beta_{\mathrm{mh}}=\frac{1}{2} \sqrt{\left(\frac{v_{\mathrm{m}}}{\rho_{\mathrm{mm}}}\right)^{2}-4 \mathrm{k}_{\mathrm{mh}}^{2} \frac{\mathrm{T}_{\mathrm{mz} \perp}}{\rho_{\mathrm{mm}}}}
\end{aligned}
$$

$A_{m x h}, B_{m x h}, A_{m y h}, B_{m y h}, A_{m h}, B_{m h}, k_{m x h}, k_{m y h}$ and $k_{m h}$ are constants, they can be obtained with boundary or initial conditions. Let:

$$
\xi_{\mathrm{mh}}=\left(\frac{v_{\mathrm{m}}}{\rho_{\mathrm{mm}}}\right)^{2}-4 \mathrm{k}_{\mathrm{mh}}^{2} \frac{\mathrm{T}_{\mathrm{mz} \perp}}{\rho_{\mathrm{mm}}}
$$

When $\xi_{\mathrm{mh}}<0$ and $\alpha_{\mathrm{mh}}>0$, Eq. 19 represents damped natural vibrations (oscillations), the frequencies are $\mathrm{f}_{\mathrm{mn}}=\beta_{\mathrm{mh}} / 2 \pi$. For cardiac cells, SRSM and CM vibrate (oscillate) under the damped mode. For a free friction approximation, $\xi_{\mathrm{mh}}<0$ and $\alpha_{\mathrm{mh}}=0$, Eq. 19 represents ideal un-damped natural vibrations, i.e., simple harmonic oscillations, the frequencies are:

$$
\mathrm{f}_{\mathrm{mn} 0}=\frac{\mathrm{k}_{\mathrm{mh}}}{2 \pi} \sqrt{\frac{\mathrm{T}_{\mathrm{m}}}{\rho_{\mathrm{mm}}}}
$$

When $\xi_{\text {mh }}>0$, Eq. 19 represents over-damped motions. When $\xi_{\mathrm{mh}}=0$, Eq. 19 represents critical damping motions. We can respectively obtain solutions for that in $\mathrm{x}$ and $\mathrm{y}$ planes by rotating $\mathrm{z}, \mathrm{x}$ and $\mathrm{y}$ in Eq. 1624. Analysis for the forced vibrations (oscillations) is the same as or similar to that for the ions in BF.

Solutions of vibrations (oscillations) for $\mathbf{C a}++\mathbf{H}+$ ATPase, Na+K+ ATPase, NCX, Ca++ carrier: Equation 5 reduces to a free (natural or homogeneous) mechanical vibration equation, after let terms at right side of the Eq. 5 be 0 . For the free vibrations (oscillations), we obtain the solution:

$$
\begin{aligned}
& \mathrm{z}_{\mathrm{sh}}(\mathrm{t})=\mathrm{A}_{\mathrm{szh}} \exp \left[\left(\alpha_{\mathrm{szh}}+\beta_{\mathrm{szh}}\right) \mathrm{t}\right]+\mathrm{B}_{\mathrm{szh}} \exp \left[\left(\alpha_{\mathrm{szh}}-\beta_{\mathrm{szh}}\right) \mathrm{t}\right] \\
& \alpha_{\mathrm{szh}}=-\frac{\mathrm{v}_{\mathrm{sz}}}{2 \mathrm{~m}_{\mathrm{sz}}} \\
& \beta_{\mathrm{szh}}=\frac{\sqrt{v_{\mathrm{sz}}{ }^{2}-4 \mathrm{~m}_{\mathrm{sz}} \mathrm{k}_{\mathrm{sz}}}}{2 \mathrm{~m}_{\mathrm{sz}}}
\end{aligned}
$$

$\mathrm{A}_{\mathrm{szh}}$ and $\mathrm{B}_{\mathrm{szh}}$ are constants and can be determined by the initial conditions. Let:

$\xi_{\mathrm{szh}}=v_{\mathrm{sz}}{ }^{2}-4 \mathrm{~m}_{\mathrm{sz}} \mathrm{k}_{\mathrm{sz}}$

When $\xi_{\mathrm{szh}}<0$ and $\alpha_{\mathrm{szh}}>0$, Eq. 25 represents damped natural vibrations, the frequencies are $\mathrm{f}_{\mathrm{szn}}=\beta_{\mathrm{szh}} / 2 \pi$. For cardiac cells and their SR, the components vibrate (oscillate) under the damped mode. For a free friction approximation, $\xi_{\mathrm{szh}}<0$ and $\alpha_{\mathrm{szh}}=0$, Eq. 25 represents ideal un-damped natural vibrations, i.e., simple harmonic oscillations, the frequencies are:

$$
\mathrm{f}_{\mathrm{szn} 0}=\frac{1}{2 \pi} \sqrt{\frac{\mathrm{k}_{\mathrm{sz}}}{\mathrm{m}_{\mathrm{sz}}}}
$$

We believe, for SANC, AVNC, Purkinje fibers and their SR, the components could naturally vibrate (oscillate) near the un-damped mode under some conditions. When $\xi_{\text {szh }}>0$, Eq. 25 represents overdamped motions. When $\xi_{\text {szh }}=0$, Eq. 25 represents critical damping motions.

From Eq. 25-29, we can obtain vibrations (oscillations) solutions, in $\mathrm{r}$ direction by changing $\mathrm{z}$ to $\mathrm{r}$ and in $\theta$ direction by changing $\mathrm{z}$ to $\theta, \mathrm{m}$ to $\mathrm{I}$; and we can also respectively obtain solutions for that in $\mathrm{x}$ and $\mathrm{y}$ planes by rotating $\mathrm{z}, \mathrm{x}$ and $\mathrm{y}$. Analysis for the forced vibrations (oscillations) is the same as or similar to that for the ions in BF. 
Solutions of myosin head vibrations (oscillations): For the free (natural) vibrations (oscillations), they are the same as or similar to solutions of free $\mathrm{Ca}++\mathrm{H}+$ ATPase vibrations (oscillations), but the solutions are over-damped motions in the real world. Analysis for the forced vibrations (oscillations) is the same as or similar to that for the ions in $\mathrm{BF}$.

\section{DISCUSSION}

We believe the principles of our models are suitable to describe how biological components or units vibrate (oscillate), wave or move, such as, the skeleton muscle contraction, the $\mathrm{Ca}++$ oscillation in neurons ${ }^{[18]}$, the flagellated sperm swimming and the Nematodes crawling $^{[3]}$. Our previous research demonstrated that the electromagnetic force could act on the cellular membrane during amitosis and mitosis ${ }^{[26]}$. The force could produce transverse membrane waves based on our model Eq. 4; our modeling results qualitatively fit a published experimental observation of oscillation waves on the cell membrane ${ }^{[12]}$.

\section{CONCLUSION}

We systematically provide new insights of automation (ignition and maintain), transportation, propagation and orientation of the cardiac cellular and sub-cellular vibrations (oscillations) and resonances, with our BioChemInfoPhysics models of momentum transfer equations.

Our modeling results imply:

- Auto-rhythmic cells (Sinoatrial Node Cells (SANC), Atrioventricular Node Cells (AVNC), Purkinje fibers), non-Auto-rhythmic ventricular myocytes and their Sarcoplasmic Reticulums (SR) work as Biological Liquid Plasma Resonators (BLPR). The resonators are biological clocks and mainly made of BF, BM and BM Transporters (BMT) that had mutually adapted and produced Biological Liquid Plasma Resonance Frequencies (BLPRF) for the resonators during their natural evolution. The resonators naturally vibrate (oscillate) near the SANC SR BLPRF that have the highest BLPRF among them. Without external stimulations, the auto-rhythmic cells and their SR can respectively vibrate (oscillate) near their BLPRF, to obtain the optimum responding amplitudes vs. internal excitation intensities: they can get the maximum biological responding amplitudes with the same excitations intensities, or they can get the same biological responding amplitudes with the minimum excitations intensities, near their BLPRF; they are frequencies selectors and select their BLPRF from disturbances frequencies to initiate the resonance. 3-D spatial vibrations (oscillations) on or across multiple BM of BLPR are temporally synchronized by the interactions (couplings) to complete cycles of the resonance of the whole resonators. Equation 1 and 4 should be considered together to obtain the actual solutions. The transverse and longitudinal vibrations (oscillations) of the membranes naturally follow that of the plasmas (free ions in BF). Vibrations (oscillations) of the biological fluids or the membranes periodically alternate the membrane structures that could correlate why or how ions channels (releasers) frequently open and close

- The electromagnetic force and the momentum of $\mathrm{Ca}++$ in ventricular myocytes also contribute to the myosin-actin motion. Some $\mathrm{Ca}++$ are bound, some $\mathrm{Ca}++$ are flowing, some $\mathrm{Ca}++$ are hitting and the all $\mathrm{Ca}++$ are pushing. One $\mathrm{Ca}++$ pushing force is roughly equal to $\mathrm{qEx}$, where $\mathrm{q}$ is an effective pushing charge of the bound $\mathrm{Ca}++$ and $\mathrm{Ex}$ is the electric field built by the flowing $\mathrm{Ca}++$, Fig. 6

- Usually, for alternating natural biological ions motions, the resistance coupling plays roles across the both fluids and membranes, the capacitance coupling plays roles only across the membranes and the inductance coupling may be ignored

- The three kinds of vibrations (oscillations): The electromagnetic, the hydrodynamic and the mechanical, can interact from each other and could be simultaneous even synchronous in a biological system.

- It is because (a) SANC SR sizes are smaller for babies than for adults, or (b) ratios of tension over mass density of cardiac SANC SRSM are higher for babies than for adults, that heart rates are higher for babies than for adults ${ }^{[11]}$. Eq. 13 and 15 or 22 and 24

\section{ACKNOWLEDGEMENT}

The researchers thank Miss Vivien Cheng for helpful suggestions and comments for this publication.

\section{REFERENCES}

1. Lloyd-Jones, D. et al., 2009. Heart disease and stroke statistics 2009 update. Circulation, 119: 480-486. DOI: 10.1161/CIRCULATIONAHA.108.191261 
2. Maltsev1, V.A., T.M. Vinogradova1, G. Edward and E.G. Lakatta1, 2006. The emergence of a general theory of the initiation and strength of the heart beat. J. Pharmacol. Sci., 100: 338-369. DOI: 10.1254/jphs.CR0060018

3. Alberts, B., A. Johnson, J. Lewis, M. Raff, K. Roberts and P. Walter, 2002. Molecular Biology of the Cell. 4th Edn., Garland Science, New York, USA., ISBN: 10: 0815332181, pp: 1616.

4. Wang, S.Q., M.D. Stern, E. R1'os and H. Cheng, 2004. The quantal nature of $\mathrm{Ca} 2+$ sparks and in situ operation of the ryanodine receptor array in cardiac cells. PNAS., 101: 3979-3984. http://www.pnas.org/content/101/11/3979.abstract

5. Yin, S., X. Zhang, C. Zhan, J. Wu and J. Cheung, 2005. Measuring single cardiac myocyte contractile force viamoving a magnetic bead. Biophys. J., 88: 1489-1495. DOI: 10.1529/biophysj.104.048157

6. Rice, J.J., M.S. Jafri and R.L. Winslow, 1999. Modeling gain and gradedness of $\mathrm{Ca}++$ release in the functional unit of the cardiac diadic space. Biophys. J., 77: 1871-1884. http://www.ncbi.nlm.nih.gov/pubmed/10512809

7. Langer, G.A. and A. Peskoff, 1996. Calcium concentration and movement in the diadic cleft space of the cardiac ventricular cell. Biophys. J., 70: 1169-1182.

http://www.pubmedcentral.nih.gov/articlerender.fc gi?artid= 1225046

8. Obara, K., N. Miyashita, C. Xu, I. Toyoshima, Y. Sugita, G. Inesi and C. Toyoshima, 2005. Structural role of countertransport revealed in $\mathrm{Ca}(2+)$ pump crystal structure in the absence of $\mathrm{Ca}(2+)$. Proc. Natl. Acad. Sci. USA., 102: 14489-14496. DOI: 10.1073_pnas.0506222102

9. Takashima, S., 2009. phosphorylation of myosin regulatory light chain by myosin light chain kinase and muscle contraction. Circulat. J., 73: 208-213. DOI: 10.1253/circj.CJ-08-1041

10. Colella, M., F. Grisan, V. Robert, J.D. Turner, A.P. Thomas and T. Pozzan, 2008. Ca2 oscillation frequency decoding in cardiac cell hypertrophy: Role of calcineurin/NFAT as Ca2_signal integrators. Proc. Natl. Acad. Sci. USA., 105: 2859-64.

http://www.pnas.org/content/105/8/2859.full.pdf+html

11. Kapit, W., R.I. Macey and E. Meisami, 1999. The Physiology Coloring Book. Murray, New York, ISBN: 10: 0321036638, pp: 320.
12. Meinhardt, H. and P.A. de Boer, 2001. Pattern formation in Escherichia coli: A model for the pole-to-pole oscillations of Min proteins and the localization of the division site. Proc. Natl. Acad. Sci. USA., 98: 14202-14207. DOI: 10.1073_pnas.251216598

13. Sweadner K.J. and C. Donnet, 2001. Structural similarities of Na,K-ATPase and SERCA, the $\mathrm{Ca}(2+)$-ATPase of the sarcoplasmic reticulum. Biochem. J., 356: 685-704. http://www.ncbi.nlm.nih.gov/pubmed/11389677

14. Lu, G., J.M. Westbrooks, A.L. Davidson and J. Chen, 2005. ATP hydrolysis is required to reset the ATPbinding cassette dimer into the resting-state conformation. Proc. Natl. Acad. Sci. USA., 102: 17969-17974. DOI: 10.1073_pnas.0506039102

15. Rigos, C.F., T.M. Nobre, M.E. Zaniquelli, R.J. Ward and P. Ciancaglini, 2008. The association of $\mathrm{Na}, \mathrm{K}-$ ATPase subunits studied by circular dichroism, surface tension and dilatational elasticity. J. Colloid Interface Sci., 325: 478-484. DOI: 10.1016/J.JCIS.2008.06.011

16. Holley, D.C. and M.P. Kavanaugh, 2008. Interactions of alkali cations with glutamate transporters. Philos Trans. R. Soc. Lond. B. Biol. Sci. DOI: $10.1098 /$ rstb.2008.0246

17. Noble, D. and A. Herchuelz, 2007. Role of $\mathrm{Na} / \mathrm{Ca}$ exchange and the plasma membrane $\mathrm{Ca} 2+-\mathrm{ATPase}$ in cell function. Conference on $\mathrm{Na} / \mathrm{Ca}$ exchange. EMBO Rep., 8: 228-32. DOI: 10.1038/sj.embor.7400914

18. Yamashita, M., 2008. Synchronous $\mathrm{Ca}(2+)$ oscillation emerges from voltage fluctuations of $\mathrm{Ca}(2+)$ stores. FEBS J., 275: 4022-4032. DOI:10.1111/j.1742-4658.2008.06543.x

19. Uman, M.A., 1964. Introduction to Plasma Physics. McGraw Hill, New York. ISBN: 10:0070657440, pp: 240.

20. Sokolnikoff, I.S. and R.M. Redheffer, 1966. Mathematics of Physics and Modern Engineering. 2nd Edn., McGraw Hill, New York, ISBN: 10: 0070596255, pp: 752.

21. Foster, K.R. and H.P. Schwan, 1986. Dielectric Propertied of Tissue. In: CRC Handbook of Biological Effects of Electromagnetic Fields, Polk, C. and E. Postow (Eds.). CRC Press, Boca Raton, Florida. ISBN: 10: 0849332656.

22. Lamb, S.H., 1945. Hydrodynamics. 6th Edn., Dover, New York, pp: 738. http://books.google.com.pk/books?id=KKsNAQA AIAAJ $\& q=$ Hydrodynamics. $\& \mathrm{dq}=$ Hydrodynamics. \&pgis $=1$ 
23. Lindsay, R.B., 1960. Mechanical Radiation. McGraw Hill, New York pp: 415. http://books.google.com.pk/books?id=q6Q8AAAA IAAJ\&q=Mechanical+Radiation\&dq=Mechanical+ Radiation\&pgis $=1$

24. Meirovitch, L., 1975. Elements of Vibration Analysis. McGraw Hill, New York, USA., ISBN: 0070413401, pp: 495.
25. Cheng, K. and C.H. Zou, 2007. Biomedicine and informatics model of alzheimer's disease. AJBB., 3: 145-149. http://www.scipub.org/fulltext/ajbb/ajbb33145-149.pdf

26. Cheng, K. and C.H. Zou, 2005. 3-D physical models of amitosis (cytokinesis). Med. Hypotheses, 64: 88-91.

DOI: 10.1016/j.mehy.2004.06.007 